\title{
DNA Barcoding of Cyprinid Fish Chagunius chagunio Hamilton,1822 from Phewa Lake, Nepal
}

\author{
Bhaba Amatya ${ }^{1}$ \\ ${ }^{1}$ Prithvi Narayan Campus, Pokhara, Tribhuvan University, Nepal \\ Correspondence: Bhaba Amatya, Prithvi Narayan Campus, Pokhara, Tribhuvan University, Nepal. Tel: \\ 98-0-654-4253. E-mail: bhabaamatya@yahoo.com
}

Received: September 1, 2019

Accepted: September 22, 2019 Online Published: September 28, 2019

doi:10.5539/ijb.v11n4p88

URL: https://doi.org/10.5539/ijb.v11n4p88

\begin{abstract}
The present study is the first of its type that uses a technique of DNA barcoding to determine identification and relationship of a species of fish from Phewa lake, Nepal. The mitochondrial DNA from two ethanol-preserved samples of fish, randomly collected from Phewa lake, was extracted using Gene AllExgene ${ }^{\mathrm{TM}}$ tissue extraction kit. 650 base pair of mitochondrial cytochrome c oxidase subunit 1 (CO1) was amplified using a cocktail of four primers and was sequenced bidirectionaly using Sanger sequence method. The DNA sequences were edited using AliView software. The sequences confirmed Chagunius chagunio as their alignment with 16 reference sequences belonging to Chagunius chagunio in the NCBI GenBank, scored highest percentage of Query Cover (75\% to 100\%) and Percentage Identity (97.29\% to 100\%). The MEGA software analysed the DNA sequences to obtain their corresponding protein sequences. The DNA sequences were submitted to the GenBank and accession numbers (MN087472 and MN087473) were obtained. Clustal Omega software analysed multiple sequence alignment among 19 homologous DNA sequences of Chagunius chagunio from India, Bangladesh and Phewa lake, Nepal. The percentage of similarity among the aligned sequences was calculated as $39.3 \%$. Based on the neighbour joining tree, the Chagunius chagunio of Phewa lake is found closely related with Chagunius chagunio of Bangladesh.
\end{abstract}

Keywords: DNA Barcode, Chagunius Chagunio, Cyprinid Fish, CO1

\section{Introduction}

The cyprinid, Chagunius chagunio, commonly called 'rewa' in Nepal, is distributed in India, Bangladesh, Pakistan, Thailand, Myanmar and Nepal. It is a least concerned species in IUCN red list status (2014). In Nepal, it is a vulnerable species of fish and is distributed in different rivers of the country, including Phewa lake, Bagmati, Trishuli, Gandaki, Bheri, Karnali and Mahakali rivers of Nepal.

In Phewa lake, the cyprinid fishes are facing steep competition with the invasive species, mainly tilapia (Oreochromis niloticus and O. mossambicus). Therefore, conservation of the cyprinid species is a special concern in the recent years. One of the best ways to protect a species is to preserve its genetic resources and genetic diversity besides the preservation of the ecosystem (Hedrick, 2001). As there is complete lack of information on genetic analysis of fish species in the lake, I have barcoded a single species of cyprinid fish from the lake using partial sequence of mitochondrial $\mathrm{CO} 1$ gene. The reasons for the selection of mitochondrial gene are: it is a haploid genome; it shows high copy number and lacks introns; it exhibits low recombination and is maternally inherited. Species identification through genetic analysis is almost always efficiently solved by the use of a standardized molecular approach, such as, the DNA barcoding, using CO1 gene (Hebert et al., 2003; Hjibabei et al., 2007). The most commonly used gene that is used as the barcoding marker, is protein coding gene cytochrome-c oxidase 1 with base length of 648 bp (Zhang \& Hewitt, 1997).

The sequence of 648 base pair of mitochondrial cytochrome c oxidase 1 gene (CO1) is used as the DNA barcode, which is highly reliable in identifying most of all animal groups. The advantage of using CO1 is that it is short enough to be sequenced quickly and cheaply yet long enough to identify variations among species. The suitability of $\mathrm{CO} 1$ gene for species identification is due to the fact that its mutation rate is often fast enough to distinguish closely related species and also because its sequences are conserved among conspecifics. High mutation rate in CO1 causes intraspecific variation which leads to species delimitation/identification (Hlaing et al., 2009; Wheat \& Watt, 2008; Williams \& Knowlton, 2001). Congeneric species of animals show more than $2 \%$ sequence 
divergence (Hebert et al., 2003). Intraspecific divergences in mitochondrial genes in animal species are rarely higher than $2 \%$ and most are less than $1 \%$ (Avise, 2000). The higher divergence occurs when the species are geographically isolated. Many causes of high divergences are due to unclear status and taxonomic uncertainty (Avise \& Walker, 1999).

Mitochondrial genomes are small (usually less than 20,000 bp), circular and maternally inherited (Boor, 1999). These genes are preferably used as universal markers in animal DNA barcoding as they are being maternal (Birky, 2001). The DNA genome is high in number per cell and are useful for population genetic and phylogenetic studies (Hu et al., 2004; McManus et al., 2004). Mitochondrial DNA is regarded as an important tool in the study of evolutionary relationships among various taxa owing to its conserved protein coding regions, high variability in non-coding sequences, and lack of recombination (Olive et al., 1983; Ingman et al., 2000). Sequence divergence accumulates more rapidly in mitochondrial DNA than in nuclear DNA owing to a faster mutation rate and lack of repair system, meaning that it often contains high levels of informative variation (Khan et al., 2008). The mitochondrial CO1 gene offers different observations in environmental science and systematic of fishes (Hubert et al., 2008) and permits researchers to receive ambiguous species to accomplish data practically and rapidly (Cowan et al., 2006).

The DNA barcodes are stored in an open-access digital library (Barcode of Life Data Systems and GenBank/National Center for Biotechnology Information) that can be used to compare the DNA barcode sequences of unidentified samples from the field by matching them to known sequences with associated species names in the database, so that users can recognize species and retrieve information about them quickly and cheaply.

The DNA barcoding of organism is effective in species identification at all stages of life, differentiating among phenotypically alike species (cryptic species) and identifying products in commerce, e.g. herbal supplements, wood, skin, bone and other animal parts .Based on the advantages of DNA barcoding, scientists all over the world have established the database and repositories of mitochondrial DNA sequences for all animals, including fish, and is adopted as a global bio-identifying system for animals in recent years.

\section{Methods and Methodology}

\subsection{Study Area}

Phewa lake is located at the south western part of kaski district about 200km west from Kathmandu Valley. The lake is at an altitude of $742 \mathrm{~m}(2,434 \mathrm{ft})$ and covers an area of about $4.43 \mathrm{~km}^{2}(1.7 \mathrm{sq} \mathrm{m})$ (Rai, 2000). It has an average depth of about $8.6 \mathrm{~m}(28 \mathrm{ft})$ and a maximum depth of $24 \mathrm{~m}$ (79ft) (Shrestha, 2003). The maximum water capacity of the lake is approximately $43,000,000$ cubic meters $(35,000$ acre $\mathrm{ft}$ ) (Pokharel, 2003). Cyprinid is the dominant fish species in Phewa lake in terms of species richness and abundance (Giri, 2013). DNA extraction, polymerase chain reaction and gel electrophoresis was carried out at the Centre for Molecular Dynamics Nepal (CMDN) in Kathmandu, Nepal.

\subsection{Sample Collection}

The fish specimens were caught in the wild and morphologically identified in situ by visual inspection. Two samples, each of approximately $100 \mathrm{mg}$ of white muscle tissue, and fin clip from two individual fish from the lake were collected and preserved in $95 \%$ ethanol.

\subsection{Mitochondrial DNA Extraction}

The DNA was extracted by using Gene AllExgene ${ }^{\mathbf{T M}}$ tissue extraction kit. The following steps were involved in the purification of DNA:

$1.20 \mathrm{mg}$ of tissue was minced with a sharp scalpel as small as possible and put in a $2 \mathrm{ml}$ tube and mixed with $200 \mathrm{ul}$ of TL buffer and vortexed for 15 seconds. (This step was for lysis of cell of the sample).

2. $20 \mathrm{ul}$ of proteinase $\mathrm{K}$ solution was added to it and mixed by vortexing. Incubation was done at $56^{\circ} \mathrm{C}$ for overnight for complete lysis. (This step was for breaking down of cell protein).

3. 400ul of buffer TB was added and mixed by vortexing. The tube was spinned down briefly to remove any drops from inside of the lid.

4. The mixture was transferred into the spin column for centrifugation for $1 \mathrm{~min}$ at $6000 \mathrm{xg}$ and replaced the collection tube with new one.

5. $600 \mathrm{ul}$ of Buffer BW was added and centrifuged for $30 \mathrm{sec}$ at $6000 \mathrm{xg}$ above and replaced the collection tube with a new one. 
6. 700ul of buffer TW was added and centrifuged for $30 \mathrm{sec}$ at $6000 \mathrm{xg}$ above. The filtrate was discarded and the SV column was reinserted back into the collection tube.

7. The mixture was centrifuged at full speed (above 13000xg) for $1 \mathrm{~min}$ to remove residual wash buffer and placed the SV column in a fresh $1.5 \mathrm{ml}$ tube.

8. $100 \mathrm{ul}$ of buffer $\mathrm{AE}$ was added and incubated for $2 \mathrm{~min}$ at room temperature and centrifuged at full speed $(>13000 \mathrm{xg})$ for $1 \mathrm{~min}$. and stored at $-20^{\circ} \mathrm{C}$.

\subsection{Amplification and Sequencing of CO1 Fragment}

The partial CO1 segment of mitochondrial DNA was targeted for DNA barcoding using cocktail of four fish specific primers (Table 1) which amplified $650 \mathrm{bp}$ region of the gene (Ivanova et al., 2007).

Table 1. Primers used for $\mathrm{CO} 1$ amplification

\begin{tabular}{|c|c|c|c|c|}
\hline Target gene & Primer ID & Sequence $5^{\prime}-3^{\prime}$ & Band size (bp) & Reference \\
\hline \multirow{4}{*}{$\mathrm{CO} 1$} & VF2_t1 & TGTAAAACGACGGCCAGTCAACCAACCACAAAGACATTGGCAC & \multirow{4}{*}{650} & \multirow{4}{*}{ Ivanova et al. (2007) } \\
\hline & FishF2_t1 & TGTAAAACGACGGCCAGTCGACTAATCATAAAGATATCGGCAC & & \\
\hline & FishR2_t1 & CAGGAAACAGCTATGACACTTCAGGGTGACCGAAGAATCAGAA & & \\
\hline & FR1d_t1 & CAGGAAACAGCTATGACACCTCAGGGTGTCCGAARAAYCARAA & & \\
\hline
\end{tabular}

A total of $25 \mu 1$ PCR final reaction was prepared containing $12.5 \mu 1$ of $2 \times$ Qiagen multiplex master mixes, $2.5 \mu 1$ of $5 \times$ Q-solution, $0.25 \mu 110 \mathrm{pMol} / \mu 1$ fish CO1 cocktail primer sets and $2 \mu 1$ of extracted DNA. The thermocycling (MJ research Tetrad PTC -225 Thermal cycler, USA) condition was $95^{\circ} \mathrm{C}$ for $15 \mathrm{~min}$ followed by touch down PCR of 5 cycles at $94^{\circ} \mathrm{C}$ for $60 \mathrm{secs}, 48^{\circ} \mathrm{C}$ for $50 \mathrm{sec}$ and $72^{\circ} \mathrm{C}$ for $50 \mathrm{sec}$ followed by 35 cycles at $94^{\circ} \mathrm{C}$ for $60 \mathrm{secs}, 50^{\circ} \mathrm{C}$ for $50 \mathrm{sec}$ and $72^{\circ} \mathrm{C}$ for $50 \mathrm{sec}$ with the final extension at $72^{\circ} \mathrm{C}$ for $5 \mathrm{~min}$. The amplified $650 \mathrm{bp}$ target PCR product was visualized in Gel-Doc, (Maor Scientific TM) under 2\% agarose gel electrophoresis. Both the samples amplified positive.

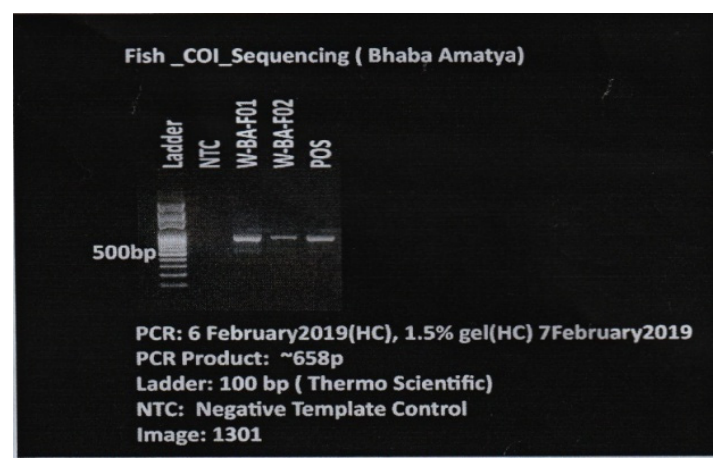

Figure 1. Cytochrome Oxidase-1 PCR run on 1.5\% agarose gel with bands appearing at approximately $650 \mathrm{bp}$ (Fish sample codes: W-BA-F01, W-BA-F02)

The amplified PCR products were purified using enzymatic clean up (ExoSAP-IT) removing unconsumed dNTPs and primers and sequencing reactions were performed in a MJ Research PTC-225 Peltier Thermal Cycler using a ABI PRISM ${ }^{\circledR}$ BigDyeTM Terminator Cycle Sequencing kits with AmpliTaq ${ }^{\circledR D N A}$ polymerase (FS enzyme) (Applied Biosystems), following the protocols supplied by the manufacturer. Single-pass sequencing was performed on each template using species specific both forward and reverse primers. The fluorescent-labeled fragments were purified from the unincorporated terminators with BigDye ${ }^{\circledR X T e r m i n a t o r}{ }^{\mathrm{TM}}$ purification protocol. The samples were re-suspended in distilled water and subjected to electrophoresis in an ABI $3730 \times 1$ sequencer (Applied Biosystems).

\subsection{Data Analysis}

The two sequence reads of each sample were processed for trimming followed by assembling via AliView software (Larsson, 2014). The conversion website Endmemo was used to calculate GC- content and the length of the sequences. To confirm the identity of the amplified sequences, I conducted BLAST (Basic Local Alignment Search Tool) searches by inputting the FASTA format of the sequences using the megablast search for highly 
similar sequences in the GenBank (https://blast.ncbi.nlm.nih.gov/Blast.cgi).The DNA sequences were submitted to GenBank to obtain accession numbers. A software program MEGA 3.1 (Molecular Evolutionary Genetics Analysis, MEGA Inc., Englewood, NJ) was used to obtain protein sequence. A new multiple sequence alignment program called Clustal Omega (EMBL-EBI, 2019) was used to align the DNA sequences of the samples with the reference sequences of the GenBank.

\title{
3. Result
}

\subsection{DNA Barcode}

FASTA format of the DNA barcodes of the fish samples from Phewa lake are given below:

>MN087472 Chagunius chagunio cytochrome oxidase subunit 1 (CO1) gene, partial cds; mitochondrial

\begin{abstract}
AGAATCAGAACAGGTGTTGATATAAAATTGGATCCCCACCCCCTGCCGGGTCGAAGAATGTGGTGTTGAGGTTACG ATCTGTTAGAAGTATTGTAATTCCTGCGGCTAAAACTGGAAGGGATAAAAGAAGCAGCACGGCAGTTACAAGCACA GATCACACAAATAAGGGTGTTTGATATTGGGAGATAGCTGGAGGTTTCATATTAATAATTGTGGTGATGAAATTAA TTGCTCCCAGAATTGATGAAACACCAGCTAAGTGTAAAGAAAAGATGGTTAGGTCGACGGATGCCCCTGCGTGGGC TAGGTTACCTGCCAGAGGCGGATATACTGTCCATCCTGTTCCGGCTCCGGCTTCAACACCAGAGGAGGCTAAAAGC AGTAAGAATGAAGGGGGTAATAGTCAAAAGCTTATATTGTTTATTCGTGGGAATGCTATATCGGGGGCTCCAATTA TGAGGGGTACTAATCAGTTTCCAAAGCCTCCAATAAGAATGGGTATAACTATAAAGAAAATTATTACGAAAGCATG GGCGGTAACGATTACATTGTAGATTTGATCGTCGCCTAGAAGTGATCCGGGTTGGCTCAGTTCGGCTCGAATGAGG AGACTTAAAGCAGTTCCTACTATCCCGGCTCAGGCACCAAATACAAGATAAAGAGTGC
\end{abstract}

[GC- content: 44\%, DNA sequence length: 666bp]

>MN087473 Chagunius chagunio cytochrome oxidase subunit 1 (CO1) gene, partial cds; mitochondrial

\begin{abstract}
CGAAGAATCAGAACAGGTGTTGATATAAAATTGGATCCCCACCCCCTGCCGGGTCGAAGAATGTGGTGTTGAGGTT ACGATCTGTTAGAAGTATTGTAATTCCTGCGGCTAAAACTGGAAGGGATAAAAGAAGCAGCACGGCAGTTACAAGC ACAGATCACACAAATAAGGGTGTTTGATATTGGGAGATAGCTGGAGGTTTCATATTAATAATTGTGGTGATGAAAT TAATTGCTCCCAGAATTGATGAAACACCAGCTAAGTGTAAAGAAAAGATGGTTAGGTCGACGGATGCCCCTGCGTG GGCTAGGTTACCTGCCAGAGGCGGATATACTGTCCATCCTGTTCCGGCTCCGGCTTCAACACCAGAGGAGGCTAAA AGCAGTAAGAATGAAGGGGGTAATAGTCAAAAGCTTATATTGTTTATTCGTGGGAATGCTATATCGGGGGCTCCAA TTATGAGGGGTACTAATCAGTTTCCAAAGCCTCCAATAAGAATGGGTATAACTATAAAGAAAATTATTACGAAAGC ATGGGCGGTAACGATTACATTGTAGATTTGATCGTCGCCTAGAAGTGATCCGGGTTGGCTCAGTTCGGCTCGAATG AGGAGACTTAAAGCAGTTCCTACTATCCCGGCTCAGGCACCAAATACAAGATAAAGAGTGCT
\end{abstract}

[GC- content: 44\%, DNA sequence length: 669bp]

The BLAST result showed significant alignment of the DNA sequence of the fish samples from Phewa lake with the 16 reference sequences of Chagunius chagunio from GenBank belonging to accession numbers: AP011373.1, JX066746.1, KF742437.1, KJ476811.1, KJ476810.1, KJ476809.1, KJ476808.1, KU667387.1, MK029815.1, KY853031.1, MG604366.1, KY290058.1, MH545566.1, MG736389.1, MH102304.1 and JN965199.1. (Table 2)

Table 2. BLAST result of the alignment of DNA sequences of the fish samples with the GenBank database

\begin{tabular}{lllll}
\hline S. No. & Query cover & E-value & Percent Identity & Scientific Name \\
\hline 1.0 & $75 \%$ to $100 \%$ & 0.0 & $97.29 \%$ to $100 \%$ & Chagunius chagunio \\
\hline
\end{tabular}

\subsection{Multiple Sequence Alignment of DNA Sequences}

Multiple Sequence Alignment among 19 homologous DNA sequences of Chagunius chagunio was carried out, among which two sequences belonging to MN087473 and MN087472 are from Phewa lake, four sequences belonging to MK572094, MH102304, MK572092 and MK572093 are from Bangladesh and the remaining are from India.

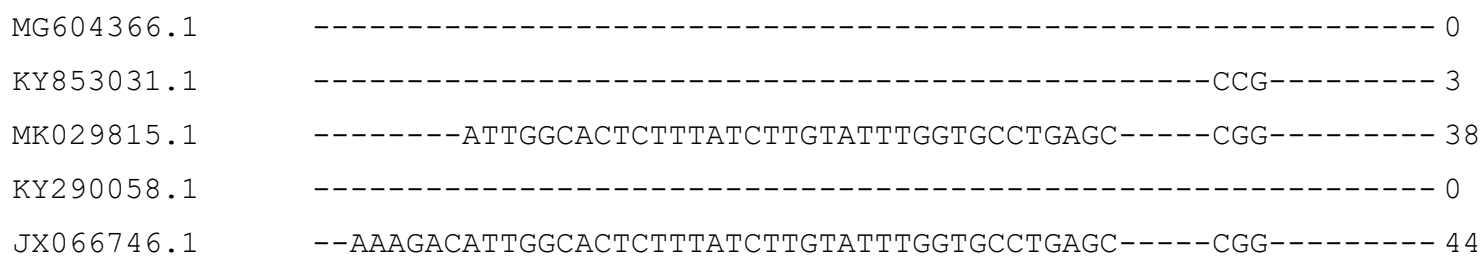


KJ476811.1

KJ 476810.1

MK572094.1

KU667387.1

MG736389.1

KJ476808.1

MK572092.1

MK572093.1

KF742437.1

JN965199.1

MH545566.1

MN0 87472.1

MN0 87473.1

MH1 02304.1

MG604366.1

KY853031.1

MK029815.1

KY290058.1

JX066746.1

KJ4 76811.1

KJ 476810.1

MK572094.1

KU667387.1

MG736389.1

KJ4 76808.1

MK572092.1

MK572093.1

KF742437.1

JN965199.1

MH5 45566.1

MN0 87472.1

MN0 87473.1

MH102304.1

MG604366.1

KY853031.1

MK029815.1

KY290058.1

JX066746.1

KJ 476811.1

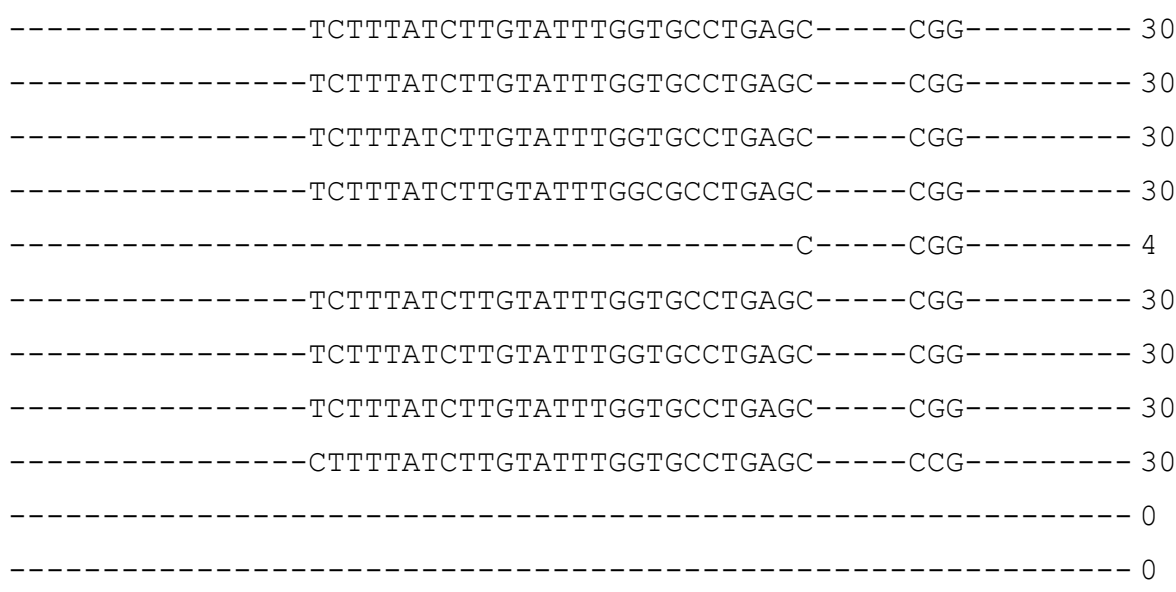

--AGAATCAGAACAgGTGTTGATATAAAATTGGATCCCCACCCCCTGCCGGGTCGAAGAA 58 GAAGAATCAGAACAGGTGTTGATATAAAATTGGATCCCCACCCCCTGCCGGGTCGAAGAA 60 CCCCCTGCCGGGTCGAAAAA 20 TGTGGTGTTGAGGTTACGATCTGTTAGAAGTATTGTAATTCCTGCGGCTAAAACTGGAAG 118 TGTGGTGTTGAGGTTACGATCTGTTAGAAGTATTGTAATTCCTGCGGCTAAAACTGGAAG 120 TGTGGTGTTGAGGTTACAATCTGTTAAAAGTATTGTAATTCCTGCGGCTAAAACTGGAAG 80

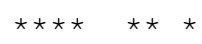

TGAGCCAACCCGGATCACTTCTAGGCGACGATCAAATCTACAATGTAATCGTTACCGCCC 100 TGAGCCAACCCGGATCACTTCTAGGCGACGATCAAATCTACAATGTAATCGTTACCGCCC 104 TGAGCCAACCCGGATCACTTCTAGGCGACGATCAAATCTACAATGTAATCGTTACCGCCC 139 TGAGCCAACCCGGATCACTTCTAGGCGACGATCAAATCTACAATGTAATCGTTACCGCCC 99 TGAGCCAACCCGGATCACTTCTAGGCGACGATCAAATCTACAATGTAATCGTTACCGCCC 145 TGAGCCAACCCGGATCACTTCTAGGCGACGATCAAATCTACAATGTAATCGTTACCGCCC 131 
KJ476810.1 MK572094.1 KU667387.1 MG736389.1 KJ476808.1 MK572092.1 MK572093.1 KF742437.1 JN965199.1 MH5 45566.1 MN0 87472.1 MN0 87473.1 MH1 02304.1

MG604366.1 KY853031.1 MK029815.1 KY290058.1 JX0 66746.1 KJ476811.1 KJ 476810.1 MK572094.1 KU667387.1 MG736389.1 KJ4 76808.1 MK572092.1 MK572093.1 KF742437.1 JN965199.1 MH5 45566.1 MN0 87472.1 MN0 87473.1 MH1 02304.1

MG604366.1 KY853031.1 MK029815.1 KY290058.1 JX066746.1 KJ4 76811.1
TGAGCCAACCCGGATCACTTCTAGGCGACGATCAAATCTACAATGTAATCGTTACCGCCC 131 TAAGCCAACCCGGATCACTTCTAGGCGACGATCAAATCTACAATGTAATCGTTACCGCCC 131 TAAGCCAACCCGGATCACTTCTAGGCGACGATCAAATCTACAATGTAATCGTTACCGCCC 131 TAAGCCAACCCGGATCACTTCTAGGCGACGATCAAATCTACAATGTAATCGTTACCGCCC 105 TAAGCCAACCCGGATCACTTCTAGGCGACGATCAAATCTACAATGTAATCGTTACCGCCC 131 TAAGCCAACCCGGATCACTTCTAGGCGACGATCAAATCTACAATGTAATCGTTACCGCCC 131 TGAGCCAACCCGGATCACTTCTAGGCGACGATCAAATCTACAATGTAATCGTTACCGCCC 131 TGAGCCAACCCGGATCACTTCTAGGCGACGATCAAATCTACAATGTAATCGTTACCGCCC 131 TGAGCCAACCCGGATCACTTCTAGGCGACGATCAAATCTACAATGTAATCGTTACCGCCC 73 TGAGCCAACCCGGATCACTTCTAGGCGACGATCAAATCTACAATGTAATCGTTACCGCCC 100 GGATAAAAGAAGCAGCACGGCAGTTACAAGCACAGATCACACAAATAAGGGTG------- 171 GGATAAAAGAAGCAGCACGGCAGTTACAAGCACAGATCACACAAATAAGGGTG-------173 GGATAAAAGAAGCAGTACGGCAGTTACAAGCACAGATCACACAAATAAGGGTG------ 133

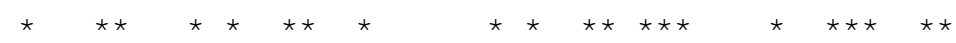

ATGCTTTCGTAATAATTTCTTTATAGTTATACCCATTCTTATTGGAGGC-TTTGGAAAC 159 ATGCTTTCGTAATAATTTTCTTTATAGTTATACCCATTCTTATTGGAGGC-TTTGGAAAC 163 ATGCTTTCGTAAтAATTTCTTTATAGTTATACCCATTCTTATTGGAGGC-TTTGGAAAC 198 ATGCTTTCGTAATAATTTCTTTATAGTTATACCCATTCTTATTGGAGGC-TTTGGAAAC 158 ATGCTTTCGTAATAATTTCTTTATAGTTATACCCATTCTTATTGGAGGC-TTTGGAAAC 204 ATGCTTTCGTAATAATTTCTTTATAGTTATACCCATTCTTATTGGAGGC-TTTGGAAAC 190 ATGCTTTCGTAAтAATTTCTTTATAGTTATACCCATTCTTATTGGAGGC-TTTGGAAAC 190 ATGCTTTCGTAATAATTTCTTTATAGTTATACCCATTCTTATTGGAGGC-TTTGGAAAC 190 ATGCTTTCGTAATAATTTCTTTATAGTTATACCCATTCTTATTGGAGGC-TTTGGAAAC 190 ATGCTTTCGTAATAATTTCTTTATAGTTATACCCATTCTTATTGGAGGC-TTTGGAAAC 164 ATGCTTTCGTAATAATTTCTTTATAGTTATACCCATTCTTATTGGAGGC-TTTGGAAAC 190 ATGCTTTCGTAATAATTTCTTTATAGTTATACCCATTCTTATTGGAGGC-TTTGGAAAC 190 ATGCTTTCGTAATAATTTCTTTATAGTTATACCCATTCTTATTGGAGGC-TTTGGAAAC 190 ATGCTTTCGTAATAATTTTCTTTATAGTTATACCCATTCTTATTGGAGGC-TTTGGAAAC 190 ATGCTTTCGTAAтAATTTCTTTATAGTTATACCCATTCTTATTGGAGGC-TTTGGAAAC 132 ATGCTTTCGTAATAATTTCTTTATAGTTATACCCATTCTTATTGGAGGC-TTTGGAAAC 159 --------TTTGATATTGGGAGATAGCTGGAGGTT-TCATATTAATAATTGTGGTGATG 221 --------TTTGATATTGGGAGATAGCTGGAGGTT-TCATATTAATAATTGTGGTGATG 223 --------TTTGATATTGGGAGATAGCTGGAGGTT-TCATATTAATAATTGTGGTGATG 183

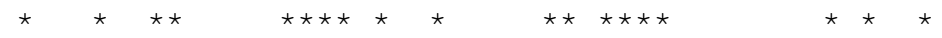
TGATTAGTACCCCTCATAATTGGAGCCCCCGATATAGCATTCCCACGAATAAACAATATA 219 TGATTAGTACCCCTCATAATTGGAGCCCCCGATATAGCATTCCCACGAATAAACAATATA 223 TGATTAGTACCCCTCATAATTGGAGCCCCCGATATAGCATTCCCACGAATAAACAATATA 258 TGATTAGTACCCCTCATAATTGGAGCCCCCGATATAGCATTCCCACGAATAAACAATATA 218 TGATTAGTACCCCTCATAATTGGAGCCCCCGATATAGCATTCCCACGAATAAACAATATA 264 TGATTAGTACCCCTCATAATTGGAGCCCCCGATATAGCATTCCCACGAATAAACAATATA 250 
KJ476810.1 MK572094.1 KU667387.1 MG736389.1 KJ4 76808.1 MK572092.1 MK572093.1 KF742437.1 JN965199.1 MH5 45566.1 MN0 87472.1 MN0 87473.1 MH1 02304.1

MG604366.1 KY853031.1 MK029815.1 KY290058.1 JX0 66746.1 KJ476811.1 KJ 476810.1 MK572094.1 KU667387.1 MG736389.1 KJ4 76808.1 MK572092.1 MK572093.1 KF742437.1 JN965199.1 MH 545566.1 MN0 87472.1 MN0 87473.1 MH1 02304.1

MG604366.1 KY853031.1 MK029815.1 KY290058.1 JX066746.1 KJ 476811.1 KJ4 76810.1
TGATTAGTACCCCTCATAATTGGAGCCCCCGATATAGCATTCCCACGAATAAACAATATA 250 TGATTAGTACCCCTCATAATTGGAGCCCCCGATATAGCATTCCCACGAATAAACAATATA 250 TGATTAGTACCCCTCATAATTGGAGCCCCCGATATAGCATTCCCACGAATAAACAATATA 250 TGATTAGTACCCCTCATAATTGGAGCCCCCGATATAGCATTCCCACGAATAAACAATATA 224 TGATTAGTACCCCTCATAATTGGAGCCCCCGATATAGCATTCCCACGAATAAACAATATA 250 TGATTAGTACCCCTCATAATTGGAGCCCCCGATATAGCATTCCCACGAATAAACAATATA 250 TGATTAGTACCCCTCATAATTGGAGCCCCCGATATAGCATTCCCACGAATAAACAATATA 250 TGATTAGTACCCCTCATAATTGGAGCCCCCGATATAGCATTCCCACGAATAAACAATATA 250 TGATTAGTACCCCTCATAATTGGAGCCCCCGATATAGCATTCCCACGAATAAACAATATA 192 TGATTAGTACCCCTCATAATTGGAGCCCCCGATATAGCATTCCCACGAATAAACAATATA 219 AAATTAATTGCTCCCAGAATTGATGAAACACC--------AGCTAAGTGTAAAGAAAAGA 273 AAATTAATTGCTCCCAGAATTGATGAAACACC--------AGCTAAGTGTAAAGAAAAGA 275 AAATTAATTGCTCCCAAAATTGATGAAACACC--------AGCTAAGTGTAAAGAAAAGA 235

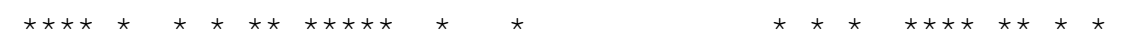

AGCTTTTGACTATTA--CCCCCTTCATTCTT------ACTGCTTTTAGCCTCCTCTGGTG 271 AGCTTTTGACTATTA--CCCCCTTCATTCTT------ACTGCTTTTAGCCTCCTCTGGTG 275 AGCTTTTGACTATTA--CCCCCTTCATTCTT------ACTGCTTTTAGCCTCCTCTGGTG 310 AGCTTTTGACTATTA--CCCCCTTCATTCTT------ACTGCTTTTAGCCTCCTCTGGTG 270 AGCTTTTGACTATTA--CCCCCTTCATTCTT------ACTGCTTTTAGCCTCCTCTGGTG 316 AGCTTTTGACTATTA--CCCССTTCATTCTT------ACTGCTTTTAGCCTCCTCTGGTG 302 AGCTTTTGACTATTA--CCCCCTTCATTCTT------ACTGCTTTTAGCCTCCTCTGGTG 302 AGCTTTTGACTATTA--CCCССTTCATTCTT------ACTGCTTTTAGCCTCCTCTGGTG 302 AGCTTTTGACTATTA--CCCCCTTCATTCTT------ACTGCTTTTAGCCTCCTCTGGTG 302 AGCTTTTGACTATTA--CCCCCTTCATTCTT------ACTGCTTTTAGCCTCCTCTGGTG 276 AGCTTTTGACTATTA--CCCCCTTCATTCTT------ACTGCTTTTAGCCTCCTCTGGTG 302 AGCTTTTGACTATTA--CCCCCTTCATTCTT------ACTGCTTTTAGCCTCCTCTGGTG 302 AGCTTTTGACTATTA--CCCCCTTCATTCTT------ACTGCTTTTAGCCTCCTCTGGTG 302 AGCTTTTGACTATTA--CCCCCTTCATTCTT------ACTGCTTTTAGCCTCCTCTGGTG 302 AGCTTTTGACTATTA--CCCCCTTCATTCTT------ACTGCTTTTAGCCTCCTCTGGTG 244 AGCTTTTGACTATTA--CCCCCTTCATTCTT------ACTGCTTTTAGCCTCCTCTGGTG 271 TGGTTAGGTCGACGGATGCCCCTGCGTGGGCTAGGTTACCTGCCAGAGGCGGATATACTG 333 TGGTTAGGTCGACGGATGCCCCTGCGTGGGCTAGGTTACCTGCCAGAGGCGGATATACTG 335 TGGTTAGGTCGACGGATGCCCCTGCGTGGGCTAGGTTACCTGCCAGAGGCGGATATACTG 295

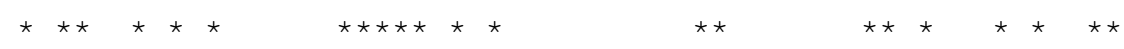

TTGAAGCCGGAGCCGGAACAGGATGGACAGTATATCCGCCTCTGGCAGGTAACCTAGCCC 331 TTGAAGCCGGAGCCGGAACAGGATGGACAGTATATCCGCCTCTGGCAGGTAACCTAGCCC 335 TTGAAGCCGGAGCCGGAACAGGATGGACAGTATATCCGCCTCTGGCAGGTAACCTAGCCC 370 TTGAAGCCGGAGCCGGAACAGGATGGACAGTATATCCGCCTCTGGCAGGTAACCTAGCCC 330 TTGAAGCCGGAGCCGGAACAGGATGGACAGTATATCCGCCTCTGGCAGGTAACCTAGCCC 376 TTGAAGCCGGAGCCGGAACAGGATGGACAGTATATCCGCCTCTGGCAGGTAACCTAGCCC 362 TTGAAGCCGGAGCCGGAACAGGATGGACAGTATATCCGCCTCTGGCAGGTAACCTAGCCC 362 
MK572094.1 KU667387.1 MG736389.1 KJ 476808.1 MK572092.1 MK572093.1 KF742437.1 JN965199.1 MH5 45566.1 MN0 87472.1 MN0 87473.1 MH1 02304.1

MG604366.1 KY853031.1 MK029815.1 KY290058.1 JX0 66746.1 KJ476811.1 KJ 476810.1 MK572094.1 KU667387.1 MG736389.1 KJ 476808.1 MK572092.1 MK572093.1 KF742437.1 JN965199.1 MH5 45566.1 MN0 87472.1 MN0 87473.1 MH102304.1

MG604366.1 KY 853031.1 MK029815.1 KY290058.1 JX066746.1 KJ4 76811.1 KJ 476810.1 MK572094.1
TTGAAGCCGGAGCTGGAACAGGATGGACAGTATATCCGCCTCTGGCAGGTAACCTAGCCC 362 TTGAAGCCGGAGCCGGAACAGGATGGACAGTATATCCGCCTCTGGCAGGTAACCTAGCCC 362 TTGAAGCCGGAGCCGGAACAGGATGGACAGTATATCCGCCTCTGGCAGGTAACCTAGCCC 336 TTGAAGCCGGAGCCGGAACAGGATGGACAGTATATCCGCCTCTGGCAGGTAACCTAGCCC 362 TTGAAGCCGGAGCCGGAACAGGATGGACAGTATATCCGCCTCTGGCAGGTAACCTAGCCC 362 TTGAAGCCGGAGCCGGAACAGGATGGACAGTATATCCGCCTTTGGCAGGTAACCTAGCCC 362 TTGAAGCCGGAGCCGGAACAGGATGGACAGTATATCCGCCTCTGGCAGGTAACCTAGCCC 362 TTGAAGCCGGAGCCGGAACAGGATGGACAGTATATCCGCCTCTGGCAGGTAACCTAGCCC 304 TTGAAGCCGGAGCCGGAACAGGATGGACAGTATATCCGCCTCTGGCAGGTAACCTAGCCC 331 TCCATCCTGTTCCGGCTCCGGCTTCAACACCAGAGGAGGCTAAAAGCAGTA------AGA 387 TCCATCCTGTTCCGGCTCCGGCTTCAACACCAGAGGAGGCTAAAAGCAGTA------AGA 389 TCCATCCTGTTCCGGCTCCGGCTTCAACACCAGAGGAGGCTAAAAGCAGTA------AAA 349

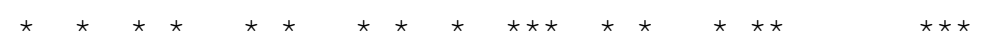
ACGCAGGGGCATCCGTCGACCTAACCATCTTTTCTTTACACTTAGCTGG--TGT------ 383 ACGCAGGGGCATCCGTCGACCTAACCATCTTTTCTTTACACTTAGCTGG--TGT------ 387 ACGCAGGGGCATCCGTCGACCTAACCATCTTTTCTTTACACTTAGCTGG--TGT------ 422 ACGCAGGGGCATCCGTCGACCTAACCATCTTTTCTTTACACTTAGCTGG--TGT------ 382 ACGCAGGGGCATCCGTCGACCTAACCATCTTTTCTTTACACTTAGCTGG--TGT------ 428 ACGCAGGGGCATCCGTCGACCTAACCATCTTTTCTTTACACTTAGCTGG--TGT------ 414 ACGCAGGGGCATCCGTCGACCTAACCATCTTTTCTTTACACTTAGCTGG--TGT------ 414 ACGCAGGGGCATCCGTCGACCTAACCATCTTTTCTTTACACTTAGCTGG--TGT------ 414 ACGCAGGGGCATCCGTCGACCTAACCATCTTTTCTTTACACTTAGCTGG--TGT------ 414 ACGCAGGGGCATCCGTCGACCTAACCATCTTTTCTTTACACTTAGCTGG--TGT------ 388 ACGCAGGGGCATCCGTCGACCTAACCATCTTTTCTTTACACTTAGCTGG--TGT------ 414 ACGCAGGGGCATCCGTCGACCTAACCATCTTTTCTTTACACTTAGCTGG--TGT------ 414 ACGCAGGGGCATCCGTCGACCTAACCATCTTTTCTTTACACTTAGCTGG--TGT------ 414 ACGCAGGGGCATCCGTCGACCTAACCATCTTTTCTTTACACTTAGCTGG--TGT------ 414 ACGCAGGGGCATCCGTCGACCTAACCATCTTTTCTTTACACTTAGCTGG--TGT------ 356 ACGCAGGGGCATCCGTCGACCTAACCATCTTTTCTTTACACTTAGCTGG--TGT------ 383 ATGAAGG--GGgtAATAGTCAAAAGCTTATATTGTTTATTCGTGGGAATGCTATATCGGG 445 ATGAAGG--GgGTAATAgTCAAAAGCTTATATTGTTTATTCGTGGGAATGCTATATCGgG 447 ATGAAGG--GgGTAATAGTCAAAAGCTTATATTGTTTATTCGTGGGAATGCTATATCGGG 407

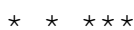
TTCATCAATTCTGGGAGCAATTAATTTCATCACCACAATTATTAATATGAAACCTCCAGC 443 TTCATCAATTTTGGGAGCAATTAATTTCATCACCACAATTATTAATATGAAACCTCCAGC 447 TTCATCAATTCTGGGAGCAATTAATTTCATCACCACAATTATTAATATGAAACCTCCAGC 482 TTCATCAATTCTGGGAGCAATTAATTTCATCACCACAATTATTAATATGAAACCTCCAGC 442 TTCATCAATTCTGGGAGCAATTAATTTCATCACCACAATTATTAATATGAAACCTCCAGC 488 TTCATCAATTCTGGGAGCAATTAATTTCATCACCACAATTATTAATATGAAACCTCCAGC 474 TTCATCAATTCTGGGAGCAATTAATTTCATCACCACAATTATTAATATGAAACCTCCAGC 474 TTCATCAATTCTGGGGGCAATTAATTTCATCACCACAATTATTAATATGAAACCTCCAGC 474 
KU667387.1 MG736389.1 KJ476808.1 MK572092.1 MK572093.1 KF742437.1 JN965199.1 MH5 45566.1 MN0 87472.1 MN087473.1 MH1 02304.1

MG604366.1 KY853031.1 MK029815.1 KY290058.1 JX0 66746.1 KJ4 76811.1 KJ 476810.1 MK572094.1 KU667387.1 MG736389.1 KJ4 76808.1 MK572092.1 MK572093.1 KF742437.1 JN965199.1 MH5 45566.1 MN0 87472.1 MN0 87473.1 MH1 02304.1

MG604366.1 KY853031.1 MK029815.1 KY290058.1 JX0 66746.1 KJ 476811.1 KJ 476810.1 MK572094.1 KU667387.1
TTCATCAATTCTGgGgGCAATTAATTTCATCACCACAATTATTAATATGAAACCTCCAGC 474 TTCATCAATTCTGGGGGCAATTAATTTCATCACCACAATTATTAATATGAAACCTCCAGC 448 TTCATCAATTCTGGGGGCAATTAATTTCATCACCACAATTATTAATATGAAACCTCCAGC 474 TTCATCAATTCTGGGGGCAATTAATTTCATCACCACAATTATTAATATGAAACCTCCAGC 474 TTCATCAATTCTGGGAGCAATTAATTTCATCACCACAATTATTAATATGAAACCTCCAGC 474 TTCATCAATTCTGGGAGCAATTAATTTCATCACCACAATTATTAATATGAAACCTCCAGC 474 TTCATCAATTCTGGGAGCAATTAATTTCATCACCACAATTATTAATATGAAACCTCCAGC 416 TTCATCAATTCTGGGAGCAATTAATTTCATCACCACAATTATTAATATGAAACCTCCAGC 443 GGCTCCAATTATGAGGGGTACTAATCAGTTTCCAAAGCCTCCAATAAGAATGGGTATAAC 505 GGCTCCAATTATGAGGGGTACTAATCAGTTTCCAAAGCCTCCAATAAGAATGGGTATAAC 507 GGCTCCAATTATGAGGGGTACTAATCAGTTTCCAAAGCCTCCAATAAAAATGGGTATAAC 467

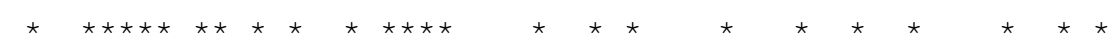

TAтСтCССААТАTСАAАC---------------ACCCTTATTTGTGTGATCTGTGCTTG 487 TATCTCCCAATATCAAAC---------------ACCCTTATTTGTGTGATCTGTGCTTG 491 TATCTCCCAATATCAAAC--------------ACCCTTATTTGTGTGATCTGTGCTTG 526 TATCTCCCAATATCAAAC---------------ACCCTTATTTGTGTGATCTGTGCTTG 486 TATCTCCCAATATCAAAC---------------ACCCTTATTTGTGTGATCTGTGCTTG 532 TATCTCCCAATATCAAAC---------------ACCCTTATTTGTGTGATCTGTGCTTG 518 TATCTCCCAATATCAAAC--------------ACCCTTATTTGTGTGATCTGTGCTTG 518 TATCTCCCAATATCAAAC---------------ACCCTTATTTGTGTGATCTGTGCTTG 518 TAтCTCCCAATATCAAAC---------------ACCCTTATTTGTGTGATCTGTGCTTG 518 TATСTCCCAATATCAAAC---------------ACCCTTATTTGTGTGATCTGTGCTTG 492 TAтСTCССАAтATCAAAC--------------ACCCTTATTTGTGTGATCTGTGCTTG 518 TATСTCCCAATATCAAAC--------------ACCCTTATTTGTGTGATCTGTGCTTG 518 TATCTCCCAAтATCAAAC---------------ACCCTTATTTGTATGATCTGTGCTTG 518 TATCTCCСАATATCAAAC---------------ACCCTTATTTGTGTGATCTGTGCTTG 518 TATCTCCCAATATCAAAC---------------ACCCTTATTTGTGTGATCTGTGCTTG 460 TATСTCCСAATATCAAAC--------------ACCCTTATTTGTGTGATCTGTGCTTG 487 TATAAAGAAAATTATTACGAAAGCATGGGCGGTAACGATTACATTGTAGATTTGATCGTC 565 TATAAAGAAAATTATTACGAAAGCATGGGCGGTAACGATTACATTGTAGATTTGATCGTC 567 TATAАAАAАAАTTATTACAAAAGCATGGGCGGTAACAATTACATTGTAAATTTGATCGTC 527

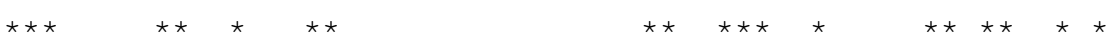
TAACTGCCGTACTGCTTCTTTTGTCCCTTCCAGTTTTAGCCGCAGGAATTACAATACTTC 547 TAACTGCCGTACTGCTTCTTTTATCCCTTCCAGTTTTAGCCGCAGGAATTACAATACTTC 551 TAACTGCCGTACTGCTTCTTTTATCCCTTCCAGTTTTAGCCGCAGGAATTACAATACTTC 586 TAACTGCCGTACTGCTTCTTTTATCCCTTCCAGTTTTAGCCGCAGGAATTACAATACTTC 546 TAACTGCCGTACTGCTTCTTTTATCCCTTCCAGTTTTAGCCGCAGGAATTACAATACTTC 592 TAACTGCCGTACTGCTTCTTTTATCCCTTCCAGTTTTAGCCGCAGGAATTACAATACTTC 578 TAACTGCCGTACTGCTTCTTTTATCCCTTCCAGTTTTAGCCGCAGGAATTACAATACTTC 578 TAACTGCCGTGCTGCTTCTTTTATCCCTTCCAGTTTTAGCCGCAGGAATTACAATACTTC 578 TAACTGCCGTGCTGCTTCTTTTATCCCTTCCAGTTTTAGCCGCAGGAATTACAATACTTC 578 
MG736389.1 KJ 476808.1 MK572092.1 MK572093.1 KF742437.1 JN965199.1 MH5 45566.1 MN0 87472.1 MN0 87473.1 MH1 02304.1

MG604366.1 KY853031.1 MK029815.1 KY290058.1 JX066746.1 KJ476811.1 KJ 476810.1 MK572094.1 KU667387.1 MG736389.1 KJ 476808.1 MK572092.1 MK572093.1 KF742437.1 JN965199.1 MH5 45566.1 MN0 87472.1 MN0 87473.1 MH102304.1

MG604366.1 KY853031.1 MK029815.1 KY290058.1 JX0 66746.1 KJ 476811.1 KJ 476810.1 MK572094.1 KU667387.1 MG736389.1

TAACTGCCGTGCTGCTTCTTTTATCCCTTCCAGTTTTAGCCGCAGGAATTACAATACTTC 552 TAACTGCCGTGCTGCTTCTTTTATCCCTTCCAGTTTTAGCCGCAGGAATTACAATACTTC 578 TAACTGCCGTGCTGCTTCTTTTATCCCTTCCAGTTTTAGCCGCAGGAATTACAATACTTC 578 TAACTGCCGTGCTGCTTCTTTTATCCCTTCCAGTTTTAGCCGCAGGAATTACAATACTTC 578 TAACTGCCGTGCTGCTTCTTTTATCCCTTCCAGTTTTAGCCGCAGGAATTACAATACTTC 578 TAACTGCCGTGCTGCTTCTTTTATCCCTTCCAGTTTTAGCC------------------ 501 TAACTGCCGTGCTGCTTCTTTTATCCCTTCCAGTTTTAGCCGCAGGAATTACAATACTTC 547 GCCTAGAAGTGATCCGGGTTGGCTCAGTTC----------GGCTCGAATGAGGAGACTTA 615 GCCTAGAAGTGATCCGGGTTGGCTCAGTTC----------GGCTCGAATGAGGAGACTTA 617 CCCTAAAAGTGATCCGGGTTGGCTCAGTTC----------GGCTCAAATGAGGAGACTTA 577

TAACAGATCGTAACCTCAACACCACATTCTTCGACCCGGCAGGGGGTGGGGATCCAATTT 607 TAACAGATCGTAACCTCAACACCACATTCTTCGACCCGGCAGGGGGTGGGGATCCAATTT 611 TAACAGATCGTAACCTCAACACCACATTCTTCGACCCGGCAGGGGGTGGGGATCCA---- 642 TAACAGATCGTAACCTCAACACCACATTCTTCGACCCGGCAGGGGGTGGGGATCCAATTT 606 TAACAGATCGTAACCTCAACACCACATTCTTCGACCCGGCAGGGGGTGGGGATCCAATTT 652 TAACAGATCGTAACCTCAACACCACATTCTTCGACCCGGCAGGGGGTGGGGATCCAATTT 638 TAACAGATCGTAACCTCAACACCACATTCTTCGACCCGGCAGGGGGTGGGGATCCAATTT 638 TAACAGATCGTAATCTCAACACCACATTCTTCGACCCGGCAGGGGGTGGGGATCCAATTT 638 TAACAGATCGTAATCTCAACACCACATTCTTCGACCCGGCAGGGGGTGGGGATCCAATTT 638 TAACAGATCGTAATCTCAACACCACATTCTTCGACCCGGCAGGGGGTGGGGATCCAATTT 612 TAACAGATCGTAATCTCAACACCACATTCTTCGACCCGGCAGGGGGTGGGGATCCAATTT 638 TAACAGATCGTAATCTCAACACCACATTCTTCGACCCGGCAGGGGGTGGGGATCCAATTT 638 TAACAGATCGTAATCTCAACACCACATTCTTCGACCCGGCAGGGGGTGGGGATCCAATTT 638 TAACAGATCGTAACCTCAACACCACATTCTTCGACCCGGCAGGGGGTGGGGATCCAATTT 638

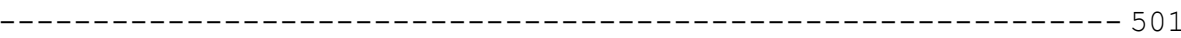
TAACAGATCGTAACCTCAACACCACATTCTTCGACCCGGCAGGGGGTGGGGATCCAATT- 606 AAGCAGTTCCTACTATCC----------------CGGCTCAGGCACCAAATACAAGAT 657 AAGCAGTTCCTACTATCC---------------_GGCTCAGGCACCAAATACAAGAT 659 AAGCAGTTCCTACTATTC---------------CGGCTCAGGCACCAAATACAAAAT 619

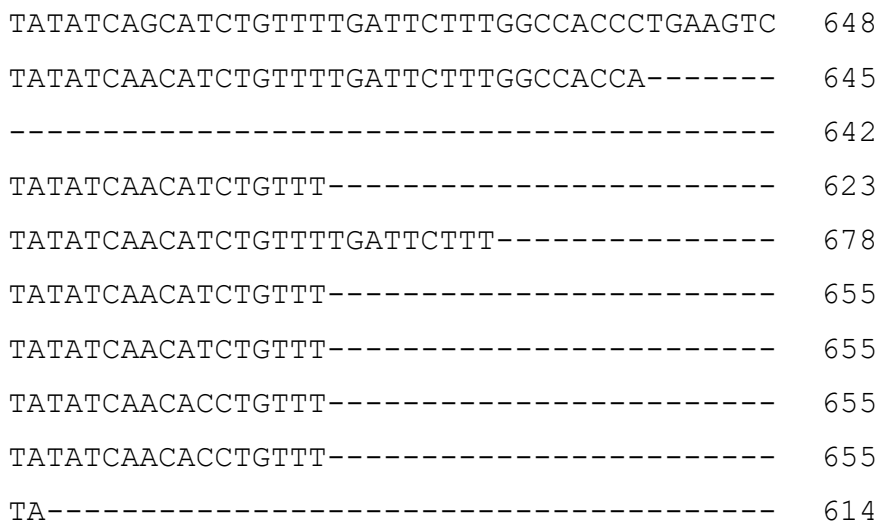




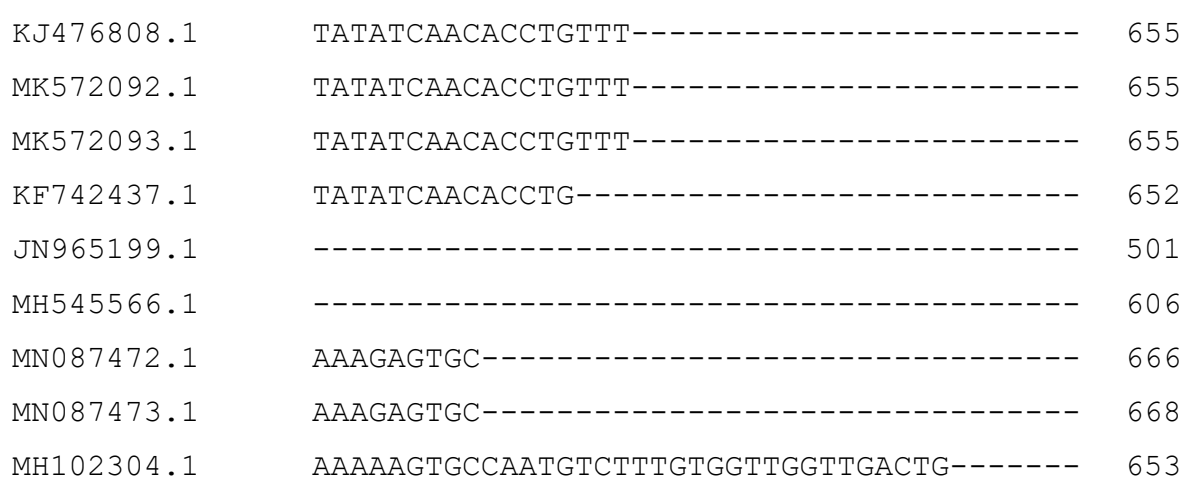

Figure 2. Multiple sequence alignment of 19 homologous DNA sequences based on Clustal Omega. The stars show number of matches among the sequences

The percentage of similarity among the aligned sequences is calculated based on the following equation.

$$
\left.\begin{array}{c}
\text { Percentage of similarity }=\frac{\text { Number of matches }}{\text { Length of the shortest DNA sequence }} \times 100 \\
\text { Percentage of similarity }=\frac{197}{501} \times 100 \\
=39.3 \%
\end{array}\right\}
$$
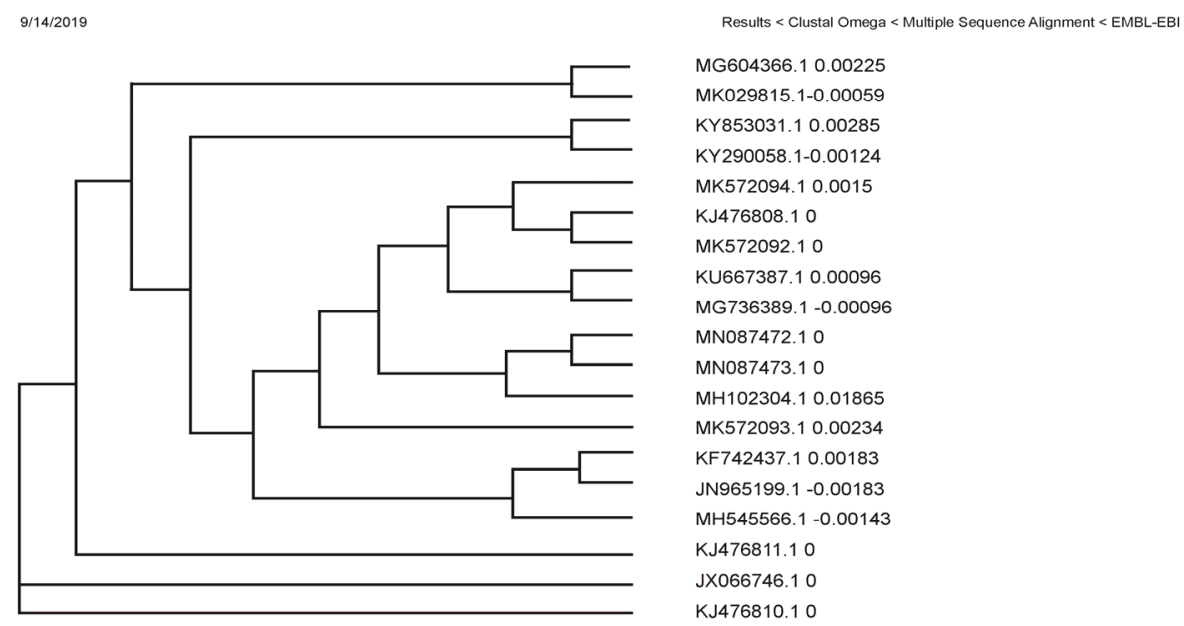

Figure 3. Multiple sequence alignment based Neighbour-Joining Tree without distance corrections

\section{Discussion}

The DNA sequence from the two samples of fish from Phewa lake scored the highest alignment scores against 16 reference sequences of Chagunius chagunio in BLAST result in terms of Query Cover $(75 \%$ to $100 \%)$ and Percentage Identity $(97.29 \%$ to $100 \%)$.The Expect Value (E-value) remained 0.0. This confirmed the identification of the samples as Chagunius chagunio. The two DNA sequences with the same percentage of GC-content and similar BLAST results provide strong evidence that the two samples of fish belong to the same species.

Sequence alignment or sequence comparison is a way of arranging biological sequences of DNA, RNA or proteins in order to distinguish regions of similarity which help us to determine how closely or distantly the organisms are related. Multiple sequence alignment is a sequence alignment of three or more biological sequences to find out their homology so that phylogenetic analysis can be obtained. In evolutionary biology, homology refers to any similarity between characters that is due to their shared ancestry. The homology among proteins and DNA is often concluded on the basis of sequence similarity. 
Sequence similarity and sequence identity are synonymous for nucleotide sequences. An identity of $39.3 \%$ in the present multiple sequence alignment of nucleotide sequences is highly desirable and suggests similarity of function and structure among the aligned sequences. According to NEB (2019), an identity of $25 \%$ or higher suggests the potential for similarity of function or structure of the aligned DNA sequences.

The most widely used approach to multiple sequence alignment is progressive technique that builds a hierarchical or tree diagram showing relationship between the sequences based on neighbor- joining method or UPGMA. In the multiple sequence alignment, if the species are closely related or while comparing individuals of the same species, it is better to use the DNA sequences. This is because the protein sequences are too similar and only a few results would be obtained. Based on the Neighbour-Joining tree, MN087473 and MN087472 which includes the DNA sequences of Chagunius chagunio from Phewa lake are monophyletic and they show very close relationship with MH102304 that includes Chagunius chagunio from Bangladesh.

\section{Conclusion}

The main purpose of the present study is to give identification to Chagunius chagunio of Phewa lake by barcoding its mitochondrial DNA and depositing the barcode sequence in the GenBank. There is no availability of DNA sequence of Chagunius chagunio in the GenBank database from Nepal as the molecular study of fish of Nepal is still at preliminary stage. This is the first deposition of DNA sequence of Nepalese Chagunius chagunio in the GenBank database which can act as a reference sequence for future studies.

The multiple sequence alignment of the DNA sequences show that the Chagunius chagunio of Phewa lake has close relationship with Chagunius chagunio from Bangladesh.

The DNA barcoding is expensive and takes nearly three months for the completion of the process including submission to the GenBank. However, it is a useful tool to quickly and accurately identify species and has the potential to prompt the discovery of new species.

\section{Acknowledgement}

I would like to thank Mrs. Jyoti Joshi and Mrs. Hemanta Chaudhary Shrestha of Centre for Molecular Dynamics Nepal (CMDN) for helping me in carrying out the barcoding of fish.

\section{Conflict of interests}

The authors declare that there is no conflict of interests regarding the publication of this paper.

\section{References}

Avise, J. C. (2000). Phylogeography: The history and formation of species. Harvard University Press, Cambridge, Massachusetts.

Avise, J. C., \& Walker, D. (1999). Species realities and numbers in sexual vertebrates: Perspectives from an asexually transmitted genome. Proc. Natl Acad. Sci., 96. 992-995. USA.

Birky, C. W. (2001). The inheritance of genes in mitochondria and chloroplasts: Laws, mechanisms, and models. Annu Rev Genet, 35, 125-48.

Boore, J. L. (1999). Animal mitochondrial genomes. Nucleic Acids Res, 27, 1767-1780.

Cowan, R. S., Chase, M. W., Kress, W. J., \& Savolainen, V. (2006). 300,000 species to identify: problems, progress, and prospects in NDA barcoding of land plants. Taxon, 55, 611-616.

EMBI-EBI. (2019). European Molecular Biology Laboratory-European Bioinformatics Institute.

Giri, K. (2013). Fish Distribution Pattern and Status of Fisher Community in Phewa lake. LAP LAMBERT Academic Publishing.

Hajibabaei, M., Singer, G. A., Hebert, P. D., \& Hickey, D. A., (2007). DNA barcoding: How it complements taxonomy, molecular phylogenetics and population genetics. Trends in Genetics, 23(4), 167-172.

Hebert, P. D. N., Ratnasingam, S., \& Dewar, J. R. (2003). Barcoding animal life: Cytochrome c oxidases subunit 1 divergences among closely related species. Proc. R. Soc. Lond. B (Suppl.), 270, s96-s99.

Hedrick, P. W. (2001). Conservation genetics: Where are we now? Trends in Ecology and Evolution, 16(11), 629-636.

Hlaing, T., Tun-Lin, W., Somboon, P., Socheat, D., Setha, T., Min, S., Chang, M. S., \& Walton, C. (2009). Mitochondrial pseudogenes in the nuclear genome of Aedesaegypti mosquitoes: Implications for past and future population genetic studies. BMC Genet, 10-11. 
Hu, M., Chilton, N. B., \& Gasser, R. B. (2004). The mitochondria genomics of parasitic nematodes of socioeconomic importance :recent progress, and implications for population genetics and systematics. $A d v$ Parasitol, 56, 133-212

Hubert et al. (2008). Identifying Canadian freshwater fishes through DNA barcodes. PloS One, 3, 24-90.

Ingman, M., Kaessmann, H., Paabo, S., \& Gyllensten, V. (2000). Mitochondrial genome variation and the origin of modern humans. Nature, 408, 708-713.

Ivanova, N., Zemlak, T., Hanner, R., \& Hebert, P. (2007). Universal primer cocktails for fish DNA barcoding. Molecular Ecology Notes, 7(4), 544-548.

Khan, H. A., Arifia, A., Homaidan, A., \& AL Farhan, A. H. (2008). Application of 16s rRNA, cytochrome b and control, region dequences for understanding the phylogenetic relationships in Oryx species. Gent. Mol. Res., 7, 1392-1397.

Kumar, S., Stecher, G., Li, M., Knyaz, C., \& Tamura, K. (2018). MEGA X: Molecular Evolutionary Genetics Analysis across computing platforms. Molecular Biology and Evolution, 35, 1547-1549.

Larsson, A. (2014). AliView: A fast and lightweight alignment viewer and editor for large data sets. Bioinformatics, 30(22), 3276-3278.

NEB. (2019). The Beginners Guide to DNA sequences Alignment. New England Biolabs 240 County Road.

Olivo, D. D., Van de Walle, M. J., Laipis, P. J., \& Hauswirth, W. W. (1983). Nucleotide sequence evidence for rapid genotypic shifts in the bovine mitochondrial DNA D-loop. Nature, 306, 400-402.

Pokharel, S. (2003). Lessons from Nepal on Developing a Strategic Plan for the Integrated Lake Basin Management: Conservation of Phewa Lake of Pokhara, Nepal (PDF). International Lake Environment Committee: World Lake Database.

Rai, A. K. (2000). Evaluation of natural food for planktivorous fish in Lakes Phewa, Begnas, and Rupa in Pokhara Valley, Nepal (PDF). Limnology, 1, 81-89.

Shrestha, P. (2003). Conservation and management of Phewa Lake ecosystem, Nepal (PDF). Aquatic Ecosystem Health and Management Society (pp. 1-4).

Shrestha, T. K. (2019). Ichthyology of Nepal. B.J.Shrestha Publisher, Kathmandu, Nepal.

Ward, R. D., Zemlac, T. C., Innes, B. H., Last, P. R., \& Hebert, P. D. N. (2005). DNA barcoding Australia's fish species. Philosophical Transactions of the Royal Society B, 360, 1847-1857.

Wheat, C. W., \& Watt, W. B. (2008). A mitochondrial -DNA-based phylogeny for some evolutionary - genetic model specis of Colias butterflies (Lepidoptera, Pieridae). Mol. Phylogenet Evol, 47, 893-902.

Williams, S. T., \& Knowlton, N. (2001). Mitochondrial pseudogenes are pervasive and often indidious in the snapping shrimp Genus Alpheus. Mol Biol Evol, 18, 1484-93.

Zhang, D. X., \& Hewitt, G. M. (1997). Assessment of the universality and utility of a set of conserved mitochondrial COI primers in insects. Insect Molecular Biology, 6, 143-150.

\section{Copyrights}

Copyright for this article is retained by the author(s), with first publication rights granted to the journal.

This is an open-access article distributed under the terms and conditions of the Creative Commons Attribution license (http://creativecommons.org/licenses/by/4.0/). 